Spirale

Arts • Lettres • Sciences humaines

\title{
Philippe Lacoue-Labarthe, dire l'irrévocable
}

\section{Ginette Michaud}

Numéro 214, mai-juin 2007

URI : https://id.erudit.org/iderudit/10406ac

Aller au sommaire du numéro

Éditeur(s)

Spirale magazine culturel inc.

ISSN

0225-9044 (imprimé)

1923-3213 (numérique)

Découvrir la revue

Citer cet article

Michaud, G. (2007). Philippe Lacoue-Labarthe, dire l'irrévocable. Spirale, (214),

$46-47$. d'utilisation que vous pouvez consulter en ligne.

https://apropos.erudit.org/fr/usagers/politique-dutilisation/ 


\section{Philippe Lacoue-Labarthe, dire l'irrévocable}

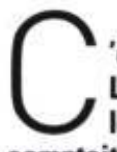

'est avec tristesse que nous avons appris la mort de Philippe Lacoue-Labarthe survenue à Paris le 28 janvier dernier. Le philosophe était venu à plusieurs reprises à Montréal et il y comptait plusieurs amis lecteurs. Spirale a régulièrement accompagné son travail, comme en témoignent les textes consacrés au fil des ans à L'imitation des modernes. Typographies II ( $\mathbf{n}^{\circ}$ 63), La poésie comme expérience $\left(n^{\circ} 66\right)$, La fiction du politique. Heidegger, l'art et la politique ( $\left.n^{\circ} 83\right)$, Le mythe nazi, avec Jean-Luc Nancy $\left(n^{\circ} 128\right)$, Poétique de I'histoire et Heidegger. La politique du poème (n' 189 ).

Le 17 février 2007 eut lieu à Paris, sous les auspices du Centre dramatique national de Montreuil et du Collège international de philosophie, une rencontre à la mémoire de Philippe Lacoue-Labarthe organisée par Jean-Christophe Bailly et Jean-Luc Nancy, à laquelle participèrent Claire Lacoue-Labarthe, Jean-Pierre Moussaron, Alain Badiou, Patrice Loraux, Leonid Karlamov, Bruno Clément, Danielle CohenLevinas, Michel Deutsch, François Martin, Rodolphe Burger, Corinne Enaudeau et Jean-Luc Nancy. Anne Alvaro y lut des textes de Philippe Lacoue-Labarthe et de Hölderlin; on y projeta Andenken, un film réalisé par Christine Baudillon avec le philosophe, et Entretiens sur I'île Saint-Pierre, un DVD de Christine Baudillon et François Lagarde (Montpellier, Hors-œil éditions); on y écouta les musiques de Beethoven, Thelonious Monk, Miles Davis et Robert Schumann. Au moment de cet Hommage, ces textes furent écrits à Montréal pour lui faire écho et saluer la pensée de Philippe Lacoue-Labarthe.

Philosophe, germaniste, professeur d'esthétique, homme de théâtre, traducteur (entre autres textes, de La naissance de la tragédie et des Fragments posthumes de Nietzsche, de l'Antigone de Sophocle de Hölderlin, des Romantiques allemands et de Walter Benjamin), écrivain. Philippe Lacoue-Labarthe fut tout cela, et bien plus. Depuis le moment où, un peu avant 1968, parurent ses premiers textes dans Le Nouveau Commerce (et « Mai $68 n$, ce n'était pas n'importe quel moment pour " commencer w), tout cela l'aura requis à la fois : le politique et le poème (la politique $d u$ poème plutôt, inlassablement, minutieusement interrogée à travers Heidegger, Hölderlin et Celan); les rapports divisés mais inséparables, contagieux et contaminés entre logos et muthos, entre la vérité et la fable, la fiction (tout récit supposé véridique, dans le mythe ou le politique); la question de la mimésis que, plus que tout autre, il aura rendue à son inimitable mimésis originaire lla conférence qu'il prononça l'an dernier au colloque "Déconstruction miméti$q^{2} e^{2}$ consacré à son travail en témoigne encore, intitulée de manière si appropriée, justement appropriée pour cerner cet inappropriable : " Mime de rien $w$, mettant ainsi * en scène $»$ la question qui lui demeura sans doute la plus « fondamentale $n$, telle que la reformule Jean-Luc Nancy dans Un commencement: "Comment un sujet aura-t-il pu se reconnaître dans son antériorité inidentifiable? » $A, 147$ ): la posée en termes de désistement et d'écho, de résonance, de retentissement, d'autobiographie précisément entendue par lui comme "mélodie obsédante n; le théâtre et toutes ses scènes, les impossibles à représenter surtout, les « primitives n et les agoniques, interminables comme le deuil: et enfin, ou au commencement de tout, la musique, le chant et la voix. les voix toujours, comme cela est si évident dans son beau recueil, Phrase (Christian Bourgois éditeur, 2000), et encore ici dans ces textes - on aurait envie de les nommer "Énigmes", mais on répugne à leur donner même ce nom - parus peu avant sa mort. où il écrit de manière si remarquable de "la différence des voix [qui] fait l'unité d'un chant d'une inconcevable tendresse " $(A, 29)$. Car, bien entendu, vaste problématique du sujet très tôt c'est toujours ce qui vient d'abord quand on pense à lui, à eux, LacoueLabarthe et Nancy, leurs voix me̊lées, croisées, séparées, si accordées. " singulières plurielles * avant la lettre, qu'on pourrait presque les croire déjà anticipées dans ce duo décrit par le jeune écrivain dans "L'un et l'autre $w: "{ }_{*} .$. deux voix distinctes se croisent, se confondent, se haussent inégalement parfois (l'une plus cristalline, l'autre plus calme), puis s'unissent à nouveau, et ainsi de suite... Mais il arrive qu'elles se taisent - qu'elles cessent non pas de résonner (elles sont bien trop faibles ou trop étouffées), mais de bruire doucement. " $(A, 63)$ Mais ces voix dédoublées, ce sont aussi déjà toutes celles du "moi ainsi constamment divisé " $(A, 104)$ de Lacoue-Labarthe seul en (ou avec) lui-même...

Une telle ouverture, une telle diversité, chaque fois traversée par le partage et la partition (dans tous les sens de ces mots) de la philosophie (disons, la pensée) et de la littérature (disons, pour en finir avec une certaine conception des belles-lettres, de l'écriture, de la " poésie pensante " $(A, 138)$, pour emprunter cette expression à Nancy). pourrait donner l'impression de ce qu'il nomme, dans l'une des proses de L'i Allégorie ", " un curieux dessin brisé ". Ce ne serait peut-être pas complètement faux - mais ce serait ne pas même commencer à suivre ce qui s'est joué dans cette œuvre de pensée qui "suivait au contraire une direction comme il l'écrit de cette figure à peine silhouettée dans " La ville désormais ", et qu'il faut justement saisir à travers ses "crochets " et ses égarements, ses éclats et ses « pointes rapides " : car " malgré donc l'illusion qu'il donnait de piétiner et de ne pouvoir progresser, c'est quand même bien le long d'un certain axe qu'il se déplaçait » $(A, 101)$.

Lui qui a si souvent parlé de "renoncement " (mais il est vrai dans un sens si particulier), il n'aura jamais rien renoncé, se chargeant des débats les plus éprouvants, héritant de Heidegger non pour le dénoncer ni le jeter, mais pour le lire au plus serré, pour tenter chaque fois de desserrer, avec rigueur et clarté, des étais logiques, entre la philosophie et le politique, entre la philosophie et le poème, et tenter d'y insuffler un peu d'air, d'espacer, de travailler au corps le plus souffrant, avec la probité, le respect, mais aussi sans piété, plutôt en gardant vive "la pitié, "la fureur de la pitié" $"(P, 64)$.

Était-ce à cause de la très haute idée qu'il se faisait de la littérature, de l'exigence de la pensée à laquelle et devant laquelle il se tenait pour lui demander des comptes, pas seulement pour s'y soumettre, exigence d'une droiture implacable qui lui enjoignait (mais pourquoi donc à lui ?) de parler toujours " le plus clairement possible „ $(P, 102)$, que ce soit en poésie ou en politique, de ce qui demeurait dans l'une et dans l'autre le plus obscur, était-ce à cause de cela qu'il était si difficile parfois de simplement s'approcher de lui? À quoi tenaient cette gêne, cette " sévère réticence "qu'il évoque encore dans l' $\alpha$ Avertissement $n$ de L'a Allégorie », même si l'on met de côté l'indéniable insuffisance de qui s'adressait à lui? Sans aucun doute en allait-il d'une impatience difficilement contenue devant l'inintelligence (les trois affects les plus à vif chez lui commencent tous par " $h$ ": "la haine, I'hébétude ou la honte "s. "forme informe du non-rapport " $P, 20)$, car il y avait bien chez lui quelque chose de coupant, de retranché. qui ne supportait aucune facilité, aucune banalité - "Simplement, il se retranchait, il refusait de se soumettre à des regards, des injonctions, à ce qui règle les rapports sous forme de réponses. „ $(P, 55)$ On sentait, toujours grondante, calmée à grandpeine, contenue mais toujours prête à sauter, conquise, comme il l'écrit dans "La scène ", mais « au prix d'un très dur travail de la volonté " $(A, 85)$ une "primitive colère " $(A, 16)$ «... ce n'est ni parce que je n'ai rien 
à dire, ni parce que j'ai envie de me taire, confiait-il dans "Phrase $I X$ ", mais parce que si je disais ce que j'ai à dire, ce ne serait pas très supportable. $(P, 72)$ En toute chose donnée, qu'elle soit de la pensée, de l'art ou de la vie, rien ne le retenait - sauf si en elle s'ouvrait soudain cela, " sans que soit jamais donné ce qui donne " $(P, 21)$. On le sentait très fortement en sa présence, on le sent aussi en le lisant : cet " impossible de dire ", mais aussi cet impossible à dire, était le fond de chacune des pensées, de chacune des paroles qui se frayaient une voie, " entre le souffle et son manque " $(A, 31)$ chez lui.

Je viens d'évoquer ce souffle coupé, ce resserrement soudain insupportable par la tension... Nous aussi, il nous arrivait de retenir notre souffle en l'écoutant, d'éprouver cette césure, ce suspens, engouffré dans la caverne ombreuse de sa voix trop profonde, l'écoutant avec angoisse, lui, comme lui-même cette voix évoquée de si près qu'elle devient la sienne propre : "Cela fait à peu près trente ans que je l'écoute, / dans la même version, toujours, où la respiration difficile de l'un des interprètes, presque / exténuée, fait que je retiens mon souffle moi-même lorsque / revient, élevé d'une octave, le motif / initial, là où précisément il a de nouveau / noté en marge : Mit innigster Empfindung. / avec ces mots qu'on croirait qu'il a lus dans Hölderlin. " (" P. XIV », dédiée " $\AA$ Jean-Luc „) Quelle émouvante mémoire de l'immémorial dans ce tracé de la voix, de ce poème daté de 1996 à la cantatrice de "L'allégorie » qui, trente ans plus tôt, " chante expire avec la provenance de la nuit " - admirable autoportrait, s'il en est... J'ajoute encore deux souffles, deux souffles à peine sur cette note: «... Nous est donné / aussi d'entendre bruire en nous, dans l'étroit de la gorge, / la douce voix que nous savons n'être pas nôtre. $\gg(P, 68)$; et encore ceci, qu'il fait dire par un de ses personnages en désistance : " la pensée, disait-il, n'est de personne, elle survient sans qu'on s'y attende ou qu'on l'ait sollicitée, c'est une tension violente mais à laquelle on s'abandonne, elle peut sembler un affaissement, il ne faut pas avoir

\section{Une pensée qui prend souffle, oui, voilà bien ce qu'était, ce que reste pour nous chaque texte, chaque livre de Lacoue-Labarthe.}

peur de laisser se dire, à peine modulé, ce qui se dit à travers nous, ce qui nous ouvre et nous traverse comme un souffle venu d'ailleurs : parce que cela doit être dit. II faudra, ensuite, pour la tirer au clair, longuement s'y acharner. " $(P, 101)$ Une pensée qui prend souffle, oui, voilà bien ce qu'était, ce que reste pour nous chaque texte, chaque livre de LacoueLabarthe.

Tendresse de l'enfance chez LacoueLabarthe : c'est par elle qu'il faut, pour finir, revenir et recommencer. Car elle aura été le commencement même pour lui, la dédicace de L' Allégorie ", qui mêle douceur et douleur, le laisse bien entendre (" Aux enfants, [...] qui auront tout de même été de très loin ma joie la plus douloureuse et mon existence la plus distinctement muette ", écrit-il). Le Stabat mater, si présent dans Phrase, la cantatrice qui ouvre tout dès l'orée de l'œuvre en chantant la mort et l'amour dans la nuit du jardin clos laissent également sourdre, à travers les figures (ce n'est plus le mot qui convient ici, tout ce travail de pensée ayant justement tenté d'en délinéer le concept) indémêlables de la mère. de l'amante, de la mort, quelque chose de cette " scène primitive ". Dans "Phrase VI *, à la question posée par l'un des interlocuteurs, "pourquoi figure-t-on le plus souvent la mort comme une femme? „, l'autre voix, la même peut-être divisée, répondait : « ce que nous reconnaissons, nous autres mort-nés, dans celle que nous reconnaissons, c'est qu'elle est précisément méconnaissable. Autrement nous l'ignorerions, elle nous resterait indifférente. Nous n'avons aucune autre raison d'aimer. Sinon de nous accompagner depuis toujours et à jamais dans notre mort, la méconnaissable immortalité. " $(P, 51)$ (Incidemment - mais cela l'est-il? - on peut remarquer, à relire tous ces textes, des « commen- cements w à la " fin ", qu'il est rarement question, même au cœur de l'intimité érotique, du sexe mais toujours du ventre, associé avec force à la phrase même : contre " le déjà donné (ventre déjà touché, phrase déjà entendue) ", le " ventre à la limite intouchable, phrase inaudible " P. 21-22.)

Comme ses amis Jacques Derrida et Jean-Luc Nancy mais autrement qu'eux, dans une tonalité plus basse encore peut-être, Lacoue-Labarthe aura gardé fidélité extrême à cette scène primitive, celle dont parle Blanchot et qu'il aura veillée jusque dans ce poème où il l'abrite peut-être. II faut savoir recueillir ce geste d'infinie tendresse où il parle de lui comme d'un autre en cet enfant qu'il est : "L'enfant, à supposer que ce soit lui, regarde. Ou bien, / peut-être, a regardé. Ce quill voit néanmoins, ce qu'il a vu, / nul ne le saura jamais, pas même lui qui l'aura / d'avance oublié mais ne cessera d'affirmer en avoir gardé / l'immémoriale mémoire : l'arrivée de rien / à ce rivage sans bord. Douceur et douleur, ensemble. $n(P, 25)$

Ce nom, Lacoue-Labarthe, je l'imagine sans peine, ne fut pas facile à porter, jusque dans sa partition intime. Devant sa noblesse, sa musicalité profonde, on était souvent tenté, pour faire court ou plus léger, moins intimidant, de l'abréger, de le couper, "Lacoue " y appelait déjà. Je ne pus jamais m'empêcher, même si j’y ai cédé aussi, de ressentir cette atteinte comme une blessure, comme si déjà il y avait dans ce nom quelque chose de trop ample pour que nous l'entendions. Seul un enfant avait su un jour en saisir toute la poésie (c'était la fille à la fine oreille de mon amie L., qui écoutait avec moi les séances si belles de ce séminaire donné par Lacoue-Labarthe à I'Université de Montréal sur Baudelaire, Mallarmé, Wagner,
Nietzsche..., tout ce que nous aimions dans, non pas dans, entre la littérature et ses autres) : en entendant ce nom si singulier, si étranger, de là-bas, résonner à ses oreilles. elle l'avait surnommé, concaténant quelques syllabes, Philou l'acrobate. (Un philosophe d'un autre genre, enfin, et qui savait danser !) Est-ce lui manquer de respect, d'affection d'oser aujourd'hui le saluer ainsi, lui le philosophe tiré en artiste? Aurait-il souri, celui qui écrivait : " Je pense à la naissance toujours innée (dégénérée) de la littérature : la diction des enfants... $(P, 17)$, d'entendre soudain si bien $\alpha$ respecter l'imprononçable,$(P, 115)$ ?

Pour Philippe, qui toujours chercha la phrase " à peine audible, / venue je ne sais d'où, d'aucune voix distinctement / prononcée / „ $(P, 129)$, la phrase qui devait sourdre.

... laisse, oui, laisse vieillir en toi et décliner / ce qui n'a pas eu lieu :/ nous y sommes tenus, contraints, de même qu'à/

l'irrévocable qui, l'un à jamais selon / l'autre, nous sépare, l'un à part l'autre nous lie

(x Phrase | x)

1. Ont paru dans l'a Allegorie * cet automne, trente-huit ans plus tard donc, des textes qui devaient être publiés à ce moment, mais qui ne le furent pas à cause des événements de Mai 68 . Ces proses d'une sôreté de trait remarquable permettent de mieux mesurer la fermeté des enjeux poétiques et philosophiques déjà au cceur des préoccupations de Lacoue-Labarthe, et qui allaient se déployer dans un registre plus spécifiquement philosophique " plus tard *, comme on dit, en tenant encore à cette conception du temps ou de la biographie. Les citations portant le sigle A proviennent de L'e Allégorie „ (Paris, Galilée * Lignes fictives », 2006), celles portant le sigle P de Phrase (Paris, Christian Bourgois éditeur, * Détroits *, 2000)

2. On peut écouter les interventions faites à ce colloque organisé par l'association * La chute dans la vallée *, qui eut lieu en janvier 2006 à Paris, autour du travail de Philippe Lacoue-Labarthe et en sa présence, en consultant le site : chttp:/ lacouelabarthe lachutedanslavallee.info/ sound/s. 